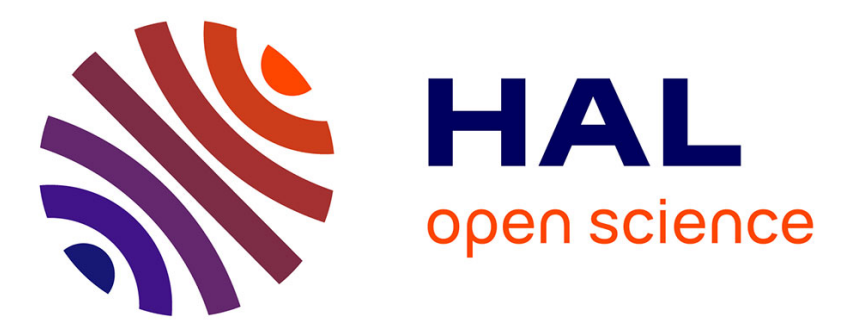

\title{
Self-assemblies of magnetic nanoparticles and di-block copolymers: Magnetic micelles and vesicles
}

\author{
Sébastien Lecommandoux, Olivier Sandre, Frédéric Chécot, Juan
}

Rodriguez-Hernandez, Régine Perzynski

\section{- To cite this version:}

Sébastien Lecommandoux, Olivier Sandre, Frédéric Chécot, Juan Rodriguez-Hernandez, Régine Perzynski. Self-assemblies of magnetic nanoparticles and di-block copolymers: Magnetic micelles and vesicles. Journal of Magnetism and Magnetic Materials, 2006, 300 (1 Sp. Iss. SI), pp.71-74. 10.1016/j.jmmm.2005.10.035 . hal-00360731

\section{HAL Id: hal-00360731 \\ https://hal.science/hal-00360731}

Submitted on 15 Nov 2018

HAL is a multi-disciplinary open access archive for the deposit and dissemination of scientific research documents, whether they are published or not. The documents may come from teaching and research institutions in France or abroad, or from public or private research centers.
L'archive ouverte pluridisciplinaire HAL, est destinée au dépôt et à la diffusion de documents scientifiques de niveau recherche, publiés ou non, émanant des établissements d'enseignement et de recherche français ou étrangers, des laboratoires publics ou privés. 


\title{
Self-assemblies of magnetic nanoparticles and di-block copolymers : magnetic micelles and vesicles
}

\author{
S. Lecommandoux, ${ }^{a}$ O. Sandre, ${ }^{b}$ F. Chécot, ${ }^{a}$ J. Rodriguez-Hernandez, ${ }^{a}$ R. Perzynski ${ }^{b *}$ \\ ${ }^{a}$ Lab. Chimie des Polymères Organiques - UMR 5629 CNRS-ENSCPB Univ. Bordeaux 1, 16 Av. Pey Berland, 33607 Pessac France \\ ${ }^{b}$ Lab. Liquides Ioniques et Interfaces Chargées - UMR 7612 CNRS, Univ. Paris 6 - case 63, 4 Place Jussieu 75252 Paris cedex 05, France
}

Elsevier use only: Received date here; revised date here; accepted date here

\begin{abstract}
Magnetic nano-composites are obtained by the self-assembly in water of polypeptide based diblock copolymers polybutadiene- $b$-poly(glutamic acid) combined with hydrophobic $\gamma-\mathrm{Fe}_{2} \mathrm{O}_{3}$ nanoparticles. These hybrid supramolecular objects are either - (3-d) spherical micelles filled with a hydrophobic ferrofluid at a concentration as high as 45 vol \% or - hollow vesicles with a (2-d) magnetic membrane. In this last case the organic amphiphilic copolymers are able to confine the hydrophobic nanoparticles within the thin layer of PB blocks. We probe these objects by AFM, by Small Angle Neutron Scattering (SANS) and by ligth scattering. Furthermore, anisotropic SANS data bring the experimental evidence of the capability to modify the shape of the mineralized membranes in response to a magnetic field intensity as low as $290 \mathrm{G}$.
\end{abstract}

Keywords: Colloids, nano-composites, block copolymers, ferrofluids, self-assembly

\section{Introduction}

To introduce inorganic nano-objects in self-organized structures of amphiphile molecules is still a challenge for physico-chemists, even if such hybrids are oftenly encountered in Nature. For example, calcium carbonates [1] and iron oxides [2,3] are colloidal particles commonly observed in organisms where they are often confined in vesicles. In addition to the biomimetic aspect of this kind of structures, the formation of hybrids constituted of nanoscale inorganic compounds dispersed in a selfassembled organic structure may lead to smart materials with specific properties brought by the inorganic nanomaterial. The challenge is here to introduce magnetic nanoparticles in self-assemblies of di-block copolymers in order to generate new supramolecular structures sensitive to an applied magnetic field. If these supramolecular objects are also able to respond to stimuli such as $\mathrm{pH}$ and ionic strength due to the presence of some polypeptide block, they form what can be called multi-responsive nanocapsules. These superparamagnetic self-assembled hybrids may offer attractive potentialities in biomedicine and biotechnology due to their dimensions $(0.1-0.5 \mu \mathrm{m})$ small enough to stay for some time in the blood circulation. Because of the properties brought by the iron oxide nanoparticles, a manipulation by an external magnetic field gradient, a local heating by a radio-frequency field for cancer radio-therapy, a labeling of organs to enhance the contrast in Magnetic Resonant Imaging become possible.

A novel type of hybrid colloids, based on the association of several polymeric systems and ferrofluids is presented here [4]. We use inorganic nanoparticles made of a magnetic iron oxide that respond to a magnetic field of low intensity. The organic part is made of mesoscopic structures (micelles or vesicles) self-assembled from amphiphilic polybutadiene-block-poly(glutamic acid) (PB$b$-PGA) diblock coplymers. These PB- $b$-PGA copolymers bearing a rod-coil structure with a cross-linkable hydrophobic block and a hydrophilic peptidic block have been synthesized recently [5]. Due to a helix-coil transition of the PGA block, such peptosomes behave as stimuliresponsive capsules; they respond to a $\mathrm{pH}$ change by a variation of their hydrodynamic diameter as large as $50 \%$ $[5,6,7]$. The magnetic nanoparticles come from colloidal suspensions of nanometric magnetic grains made of

\footnotetext{
${ }^{*}$ Corresponding author. Tel.: 33-1-4427-3267; fax: 33-1-4427-3882; e-mail: regine.perzynski@sorbonne-universite.fr
} 
maghemite $\left(\gamma-\mathrm{Fe}_{2} \mathrm{O}_{3}\right)$ and stabilized either by electrostatic charges in aqueous media [8] or by appropriate tensioactives in organic solvents [9]. Performing smallangle neutron scattering (SANS) experiments in LLB-CEA Saclay-France on PACE and PAXY spectrometers, we use these inorganic nanoparticles, embedded in supramolecular objects formed by diblock copolymers, as structural probes of the hybrid objects. Benefiting from the strong contrast of iron oxide with respect to the polymer and the solvent, we measure the structure factor $S_{\text {intra }}(q)$ of the nanoparticles inside the objects. The shape of $S_{\text {intra }}(q)$ reflects the interactions between the nanoparticles inside the object [10]. In addition at low scattering vectors $q, S_{\text {intra }}(q)$ can be associated to the global form factor $\mathrm{P}(\mathrm{q})$ of the object that they decorate $[11,12]$. The synthesized objects are also probed by static (SLS) and dynamic (DLS) light scattering and by AFM.

\section{Magnetic micelles}

We obtain the magnetic micelles mixing in water (or in a mixture of $\mathrm{H}_{2} \mathrm{O} / \mathrm{D}_{2} \mathrm{O}$ ) either $\mathrm{PB}_{48}-b-\mathrm{PGA}_{114}$ or $\mathrm{PB}_{48}-b$ $\mathrm{PGA}_{145}$ di-block copolymers with a ferrofluid based on $\gamma$ $\mathrm{Fe}_{2} \mathrm{O}_{3}$ nanoparticles.

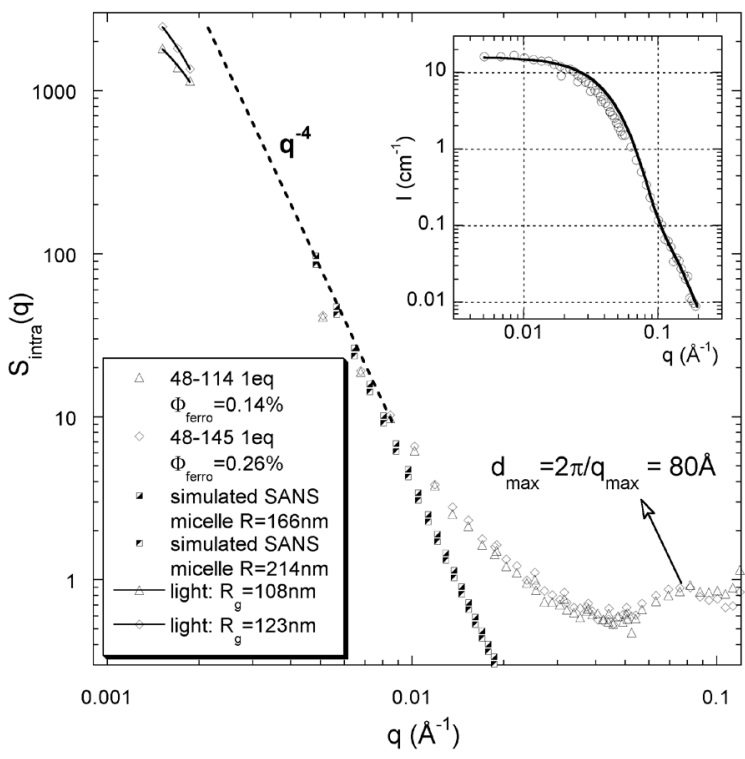

Figure $1-S_{\text {intra }}(q)$ of the hydrophobic nanoparticles confined in micelles of $\mathrm{PB}_{48}-b-\mathrm{PGA}_{114}$ and $\mathrm{PB}_{48}-b-\mathrm{PGA}_{145}$ obtained by SANS. The dashed line corresponds to a slope -4 . The points at low $q$ are obtained by SLS. Inset : SANS intensity as a function of $\mathrm{q}$ from the dilute ferrofluid, fitted with the form factor of the nanoparticles.

The nanoparticles are dispersed in dichloromethane and stabilized by a commercial phosphoric di-ester tensioactive Beycostat $\mathrm{NE}^{\circledR}$. The inset of figure 1 shows the form factor of the nanoparticles as obtained by SANS, a fit by a lognormal distribution of the diameter $d$ of nanoparticle gives $\mathrm{d}_{\mathrm{o}}=7.6 \mathrm{~nm}\left(\ln \mathrm{d}_{\mathrm{o}}=<\operatorname{lnd}>\right)$ with a standard deviation of $\ln \mathrm{d}$ $\sigma=0.25$.

The dispersions of the synthesized objects are stable in time. Combined with one mass equivalent of ferrofluid, both di-block copolymers give rise to fully dispersed suspensions. Figure 1 shows the structure factor $S_{\text {intra }}(q)$ of the nanoparticles inside the synthesized objects as obtained by SANS (the solvent is contrast matched to polymer) and SLS. At intermediate scattering vectors $\mathrm{q}, \mathrm{S}_{\text {intra }}(\mathrm{q}) \propto \mathrm{q}^{-4}$ it expresses that the nanoparticles are densely packed inside the hybrid objects. The SLS measurements lead to a radius of gyration $\mathrm{R}_{\mathrm{g}}$ of the objects of the order of $100 \mathrm{~nm}$. It is smaller than (half of) the hydrodynamic diameter $\mathrm{d}_{\mathrm{H}}$ (determined by DLS) which ranges between 225 to $430 \mathrm{~nm}$. There is a several-fold increase of hydrodynamic diameter with respect to unloaded micelles obtained without adding any ferrofluid $\left(\mathrm{d}_{\mathrm{H}} \approx 60\right.$ to $\left.70 \mathrm{~nm}\right)$. It means that the presence of the nanoparticles significantly modifies the self-assembly of di-blocks.
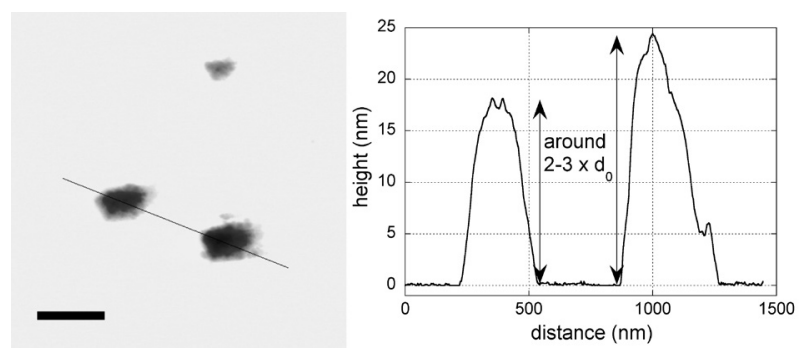

Figure 2 - left : AFM picture of $\mathrm{PB}_{48}-b$ - $\mathrm{PGA}_{114}$ copolymer micelles loaded with nanoparticles; scale bar : $400 \mathrm{~nm}$. right : Height profile along the cross-section of the AFM picture.

The ratio $2 \mathrm{R}_{\mathrm{g}} / \mathrm{d}_{\mathrm{H}}$ of the hydrid objects is found between 0.6 and 0.8 . It is close to the hard sphere value $(3 / 5)^{0.5} \approx$ 0.77. Moreover $S_{\text {intra }}(q)$ presents a correlation bump around $810^{-2} \AA^{-1}$ (see figure 1) which is associated to a most probable interparticle distance $\mathrm{d}_{\max } \approx 8 \mathrm{~nm}$ and a volume fraction of the order of $45 \%$. This high encapsulation yield, together with the global three dimensional shape of the objects is confirmed by AFM images (see figure 2). The nanoparticles appear densely packed and confined in thick bags of lateral extension comparable to the diameter $d_{H}$ determined by DLS.

We conclude that we obtain 3D-micelles bulky loaded with nanoparticles with a number of particles per micelle that can be evaluated to a few $10^{4}$. It is two orders of magnitude larger than in aggregates of magnetic nanoparticles obtained by the addition of coagulating homopolymers $[13,14]$ or of other self-assembling diblock copolymers [15]. 


\section{Magnetic vesicles in zero field}

We obtain the magnetic vesicles mixing in water (or in a mixture of $\left.\mathrm{H}_{2} \mathrm{O} / \mathrm{D}_{2} \mathrm{O}\right) \mathrm{PB}_{48}-b$ - $\mathrm{PGA}_{56}$ di-block copolymers with the previous ferofluid in dichloromethane. The structure factor of the hybrid objects is presented in figure 3. In the low q regime, $S_{\text {intra }}(q)$ approximately follows a power law $\mathrm{q}^{-2}$, which is completely different from the $\mathrm{q}^{-4}$ behavior of figure 1 . This $\mathrm{q}^{-2}$ regime could be associated to 3D aggregation of the nanoparticles under DLA or RLA processes. However AFM pictures (inset of figure 3 and figure 4) do not correspond to such a fractal cluster formation [16] nor to clusters induced by other (co)polymers $[14,17,18]$. We observe closed loops around a central hole looking like pieces of lace. These holey shapes are reproducible (see inset of figure 3 ).

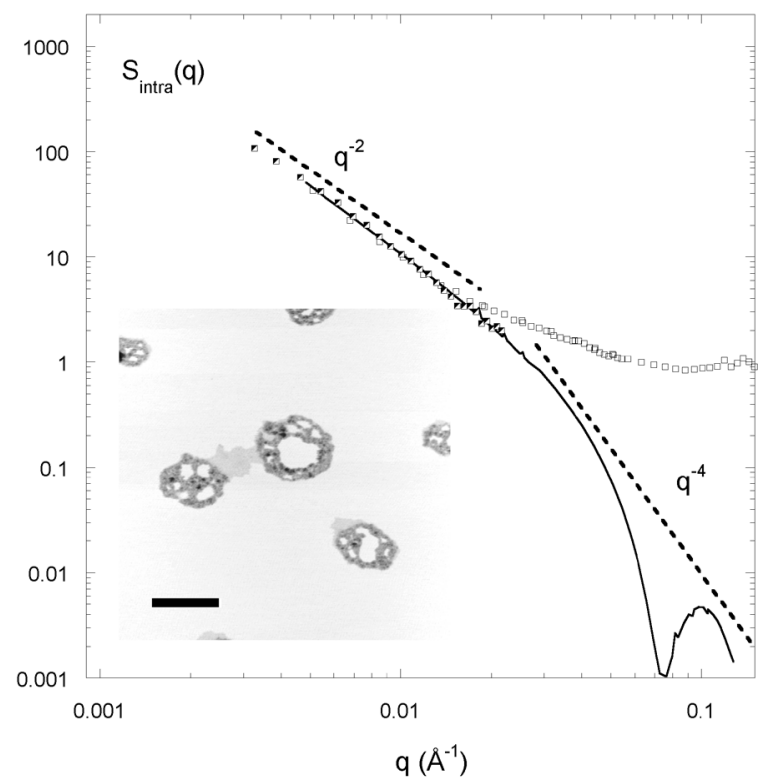

Figure 3- $S_{\text {intra }}(q)$ of the hydrophobic nanoparticles confined in micelles of $\mathrm{PB}_{48}-b-\mathrm{PGA}_{56}$ obtained by SANS. The full line corresponds to the form factor of a vesicle of radius $312 \mathrm{~nm}$ and $\delta$ $=8.7 \mathrm{~nm}$ Inset : AFM picture of dispersion of these hollow hybrid objects, the bar length is $500 \mathrm{~nm}$.
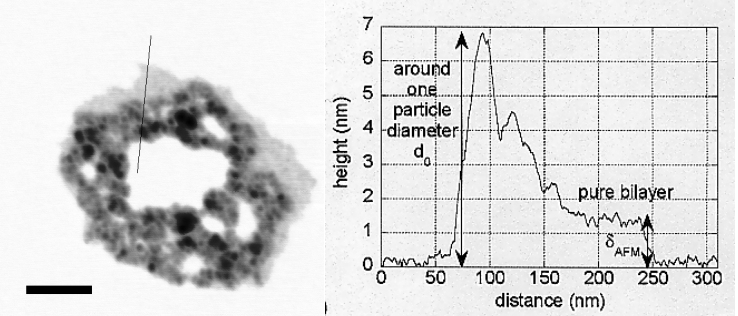

Figure 4 - left : typical AFM lace shape of $\mathrm{PB}_{48}-b-\mathrm{PGA}_{56}$ copolymer vesicles, the membrane being loaded with nanoparticles; scale bar : $150 \mathrm{~nm}$. right : Height profile along the cross-section of the AFM picture.
Presumably, because of the strong adhesion of the softs shells on the freshly cleaved surface of mica, the vesicles open pores. It leads to a ripping of the bilayers and then allows the spreading of the initially curved membranes onto the strongly adhesive substrate.

Moreover here the ratio $2 \mathrm{R}_{\mathrm{g}} / \mathrm{d}_{\mathrm{H}}$ is close to 1 which is the theoretical value for a hollow sphere. From the SANS spectra, one can also deduce the thickness $\delta$ of the layer of nanoparticles inside the membrane of the vesicle. Using the Kratky-Porod approximation [19] of the form factor of a flat infinite sheet of thickness $\delta$ in this q-region, it comes : $\ln \left(\mathrm{q}^{2} \mathrm{~S}_{\text {intra }}\right) \approx \ln \left(\mathrm{I}_{\mathrm{o}}\right)-\mathrm{q}^{2} \delta^{2} / 12, \mathrm{I}_{\mathrm{o}}$ being proportional to the surface area. For one mass equivalent of ferrofluid and in a contrast matched experiment, we get $\delta=8.6 \mathrm{~nm}$; in $\mathrm{H}_{2} 0$ due to an additional contribution of the copolymer, we obtain $\delta=10 \mathrm{~nm}$ (see further on inset of figure 6). We can conclude that in these conditions the membrane contains at most one layer of magnetic particles. Indeed we can also adjust (see figure 3) $\mathrm{S}_{\text {intra }}$ (q) at low q to the form factor of vesicles of radius $\mathrm{d}_{\mathrm{H}} / 2$ and membrane thickness $\delta$. Let us note also that $\mathrm{S}_{\text {intra }}(\mathrm{q})$ does not present any correlation bump at large $\mathrm{q}$ which confirms that the inorganic nanoparticles form, at most, one monolayer inside the polymeric membrane. We conclude that we observe hollow vesicles with a mineralized membrane.

\section{SANS probing of magnetic vesicles under field}

To study the response to an applied magnetic field, of vesicles of $\mathrm{PB}_{48}-b$ - $\mathrm{PGA}_{56}$ loaded with nanoparticles inside their membrane and to work in (almost) pure nuclear contrast [20], the system is dispersed in light water. When a magnetic field is applied, the scattering pattern becomes clearly anisotropic (see figure 5). In the $310^{-3}-210^{-2} \AA^{-1}$ q-range the lines of iso-intensity are ellipses elongated perpendicularly to the field direction, with aspect ratios increasing up to 1.3 as the field intensity reaches $1050 \mathrm{Oe}$, almost independently of the value of $q$.

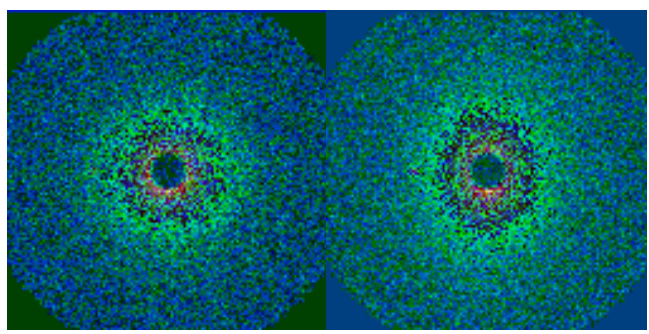

Figure 5 - SANS pattern of hollow magnetic shells in the range $310^{-3}-210^{-2} \AA^{-1}$. left : in zero field; right : under a horizontal field $\mathrm{H}=1050$ Oe.

In the SANS window of $\mathrm{q}$ which is here explored, the measurements lie far above the Guinier range and give 
information at the length scale of the membrane thickness. In addition because of the magnetic and nuclear contrasts of the $\gamma-\mathrm{Fe}_{2} \mathrm{O}_{3}$ nanoparticles dispersed in ligth water, the anisotropy of the pattern cannot be due to their magnetic scattering and is of nuclear origin [20]. In order to study the anisotropy, the 2D-scattering patterns are averaged in angular sectors $\pm 15^{\circ}$ around the two perpendicular directions // and $\perp$ defined by the magnetic field. After dividing by the isotropic form factor of the nanoparticles, we obtain two different structure factors in the // and $\perp$ directions. They are plotted in figure 6 at $\mathrm{H}=1050 \mathrm{Oe}$ and compared the value at $\mathrm{H}=0$ Oe. The inset of figure 6 is a Kratky-Porod plot $\ln \left(\mathrm{q}^{2} \mathrm{~S}_{\text {intra }}\right)$ as a function of $\mathrm{q}^{2}$.

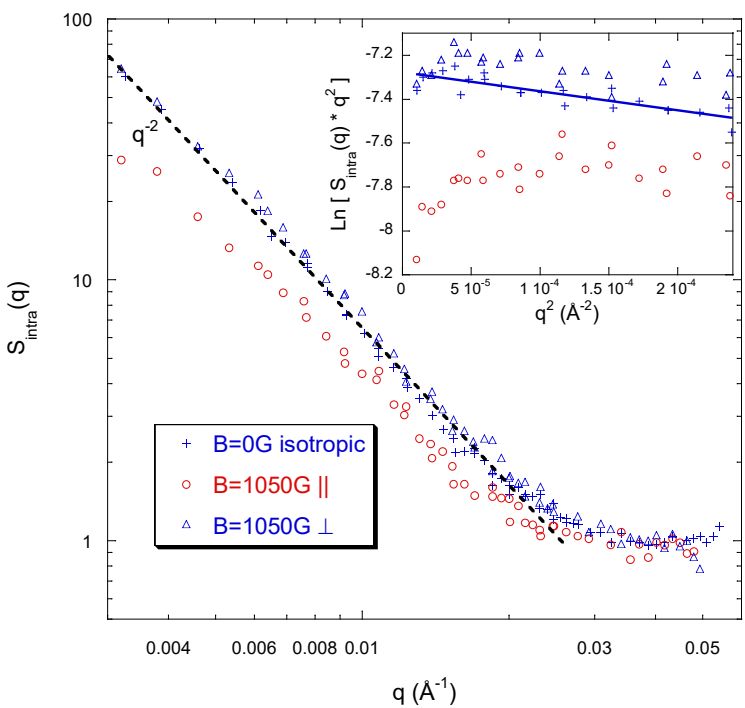

Figure 6 - Comparison between $\mathrm{S}_{\text {intra }}(\mathrm{q})$ at $\mathrm{H}=0$ and the under field determinations on sectors $\pm 15^{\circ}$ around the direction parallel to the field and around the perpendicular direction. Inset : KratkyPorod representation of the data.

Within the error bar there is no difference between the measurement in the direction perpendicular to the field and the isotropic situation at $\mathrm{H}=0$ in both representations $\mathrm{S}_{\text {intra }}(\mathrm{q})$ and $\ln \left(\mathrm{q}^{2} \mathrm{~S}_{\text {intra }}\right)=\mathrm{f}\left(\mathrm{q}^{2}\right)$. Thus the thickness of the membrane in the direction perpendicular to the field $\delta_{\perp}$ is of the same order as $\delta$, the thickness in zero field. On the contrary in the direction parallel to the field the structure factor is substantially decreased with respect to the $\mathrm{H}=0$ situation, up to $\mathrm{q}$ of the order of $210^{-2} \AA^{-1}$. We conclude that the portions of membranes mostly affected by the magnetic field are those with their normal vector parallel to the field. In that direction the Kratky-Porod representation becomes almost flat : there is an important decrease of the apparent thickness $\delta_{/ /}$associated to a deficiency of nanoparticles inside the membrane patches that cross the field direction. Thus either the membrane becomes stretched in the portions of shell crossed by the magnetic field or almost equivalently the nanoparticles move away from the magnetic poles. To our knowledge, these mineralized vesicles are the first ones to have a fluid and magnetic membrane. Other magnetic shells have been mentioned in literature $[21,22]$, but none as thin and as floppy as here.

The soft magnetic objects presented here are especially promising for drug delivery, their internal compartment being available for encapsulation of water-soluble species. A $\mathrm{pH}$ change or the application of a radio-frequency magnetic which would induce a local heating could trigger the transient opening of the envelope and the release of an encapsulated content.

Acknowledgements We thank Fabrice Cousin and Julian Oberdisse as our local contacts at the LLB facilities.

[1] S. Mann, Angew. Chem. Int. Ed. 39 (2000) 3393

[2] N.D. Chasteen, P.M. Harrison, J. Struct. Biol. 126 (1999) 182

[3] S. Ofer, I. Nowik, E.R. Bauminger, G.C. Papaefthymiou, R.B Frankel, R.P. Blakemore Biophys. J. 46 (1984) 57

[4] S. Lecommandoux, O. Sandre, F. Chécot, J. RodriguezHernandez, R. Perzynski, Adv. Mater. 17 (2005) 712

[5] F. Chécot, S. Lecommandoux, Y. Gnanou, H.-A. Klok, Angew. Chem. Int. Ed. 41 (2002) 1340

[6] F. Chécot, S. Lecommandoux, H.-A. Klok, Y. Gnanou, Eur. Phys. J. E 10 (2003) 25

[7] J. Babin, J. Rodriguez-Hernandez, S. Lecommandoux, H.-A. Klok, M.-F. Achard, Faraday Discussion 128 (2005) 179

[8] R. Massart I.E.E.E. Trans. Magn. 17 (1981) 1247

[9] S. Lefebure, E. Dubois, V. Cabuil, S. Neveu, R. Massart, J. Mater. Res. 13 (1998) 2975

[10]E. Dubois, F. Boué, V. Cabuil, R. Perzynski, J. Chem. Phys. 111 (1999) 7147

[11]J. Oderbisse, B. Demé, Macromol. 35 (2002) 4397

[12] G. Despert, J. Oderbisse, Langmuir 19 (2003) 7604

[13]S. Veintemillas-Verdaguer et al, J. Phys.D : Appl. Phys. 37 (2004) 2054

[14]D. El Kharrat, O. Sandre, P. Licinio, R. Perzynski, AIP Conf. Proc. 708 (2004) 122.

[15]M.A. Kiselev, M. Janish, P. Lesieur, A. Hoell, J. Oberdisse, G. Pepy, A.M. Kisselev, I.V. Gapienko, T. Gutberlet, V.L. Aksenov Appl. Phys. A 74 (2002) S1239.

[16]K. Butter, P.H. Bommans, P.M. Frederiks, G.J. Vroege, A.P. Philipse, Nat. Matter. 2 (2003) 88

[17]L.E. Euliss, S.G. Grancharov, S. O’Brien, T.J. Deming, G.D Stucky, C.B. Murray, G.A. Held Nano Lett. 3 (2003) 1489

[18]R.S. Prosser, S.A. Hunt, J.A. DiNatale, R.R. Vold J. Am. Chem. Soc. 118 (1996) 269

[19] O. Glatter, O. Kratky, in Small X-Ray Scattering, Academic press, New York (1983).

[20]F. Gazeau, F. Boué, E. Dubois, R. Perzynski, J. Phys. Cond. Matter. 15 (2003) S1305

[21]E.L. Bizdoacaa, M. Spasovaa, M. Farlea, M. Hilgendorff, F. Caruso, J. Mag. Mag. Mat. 240 (2002) 44

[22]D.G. Shchuki, G.B. Sukhorukov, H. Möhwald, Angew. Chem. Int. Ed. 42 (2003) 4472 